\title{
Laboratory Investigation of Early Damage Detection for an Old-Aged Reinforced Concrete Beam using Acoustic Emission and Digital Image Correlation
}

\author{
Shitong Hou ${ }^{1}$, Jianhua $\mathrm{Fan}^{2}$, Gang $\mathrm{Wu}^{3 *}$, Haochen Wang ${ }^{1}$ and Yitian Han ${ }^{1}$
}

Received 17 December 2020, accepted 9 June 2021

doi:10.3151/jact.19.700

\begin{abstract}
This paper presents an experimental study investigating the actual mechanical performance of a full-scale reinforced concrete beam that served for 60 years in an industrial environment. The primary objective is to verify early damage detection performance using acoustic emission (AE) and digital image correlation (DIC) methods based on the four-point bending test. Parameter analysis of AE provides an accurate indication of the crack opening process, especially for the early damage stage, while RA (rise time divided by amplitude) against AF (Acoustic counts divided by durable time) and b-value analyses based on AE parameters also provide specific damage propagation results for different load stages. Furthermore, owing to the good full-field measurement resolution of DIC, the strain results in each load stage more clearly present the crack pattern and distribution before the visible crack is found, and the results of the displacement derived from DIC images also capture the global and local deformation of the beam with satisfactory accuracy compared with conventional methods. A brief summary of the advantages and disadvantages of using AE and DIC for early damage detection of the full-scale beam is also provided. It is concluded that AE and DIC both exhibit good feasibility for early damage detection from the respective interior and exterior aspects. Robust signal and image processing algorithms can be integrated with these two methods for further quantitative analysis.
\end{abstract}

\section{Introduction}

Concrete structures, especially reinforced concrete structures, are the most widely used structures across the globe: their robust load-carrying capacity provides excellent reliability for transportation structures, high-rise buildings, electronic facilities, etc. These structures are initially designed with a finite intended service life, which involves enduring long-term deterioration processes. During the lifetime of a civil engineering structure, the resistance will decrease due to the external loads, extreme environments, and material degradation. To date, the primary challenge has involved the accurate assessment of the actual mechanical state of these existing structures. Infrastructure stakeholders have conducted routine and preventative maintenance methods to keep these systems functional. Visual inspection, which is the most frequently used approach, is subject to personal judgment, and several old-aged structures may be badly damaged before discovery by the inspector. Because of these drawbacks, alternative approaches have been deployed for timely inspection and evaluation.

Structural health monitoring, combined with multiple

${ }^{1} \mathrm{Ph}$.D. candidate, School of Civil Engineering, Southeast University, Nanjing, 210096, China.

${ }^{2}$ Master degree candidate, School of Civil Engineering, Southeast University, Nanjing, 210096, China.

${ }^{3}$ Professor, School of Civil Engineering, Southeast University, Nanjing, 210096, China.

*Corresponding author, E-mail: g.wu@seu.edu.cn sensing technologies, is the main method to achieve the goal of structural system evaluation. The concept of this method has existed for several years in different engineering fields (Sohn et al. 2004). This method is similar to human health inspection (Aktan et al. 2000). Displacement (Im et al. 2011; Hou and Wu 2019), acceleration (Ko and Ni 2005), and strain sensors (Torres et al. 2011) have been used for displacement and deformation monitoring. The fundamental challenge for this method is how to translate the measurement results into full-scale structural information for condition assessment (Dizaji et al. 2018). Significant research has been implemented with the development of advanced technologies and soft computing methodologies (Farrar and Lieven 2006; Vaghefi et al. 2011; Agdas et al. 2015). To date, the concept has led to more comprehensive structural evaluation, and the data management, installation cost, and interpretation of results demonstrate that SHM is still in the process of exploration of additional novel technologies. Bridges and high buildings may include sensors installed on the surface or inside the structure during the operation stage. Data processing, load capacity evaluation, and finite model updating work were conducted based on the limited response data $(\mathrm{Ou}$ and $\mathrm{Li}$ 2010). However, the installation of sensors and sensing systems leads to extra challenges including cost, access, and durability (Alipour et al. 2019), and the non-contact method meets the needs for noninvasive and flexible application. Feng (Feng and Feng 2017) validated the potential of vision displacement sensors for cost-effective structural health monitoring with high accuracy, and the dense full-filed displacement measurements can provide 
robust dynamic characteristics. Park (Park et al. 2010) proposed a novel vision-based displacement measurement using a partition approach to solve the horizontal displacement of a high-rise building structure. Though the non-contact method enables feasibility for easier measurement, the target attachment and measurement distance lead to much more noise, which affects the accuracy and related evaluation. Khuc (Khuc and Catbas 2017) provided the pixel-based measurement framework using matching algorithm, and a framework for evaluating the results of non-contact measurements is proposed. In addition to the displacement measurement, vision-based methods, especially digital image correlation (DIC), also provide full-field response across the surfaces of structures, and the specific information makes strain measurement and efficient damage detection possible. Hoult (Hoult et al. 2013) demonstrated the experimental accuracy of two-dimensional strain measurement for small structural specimens. Carroll (Carroll et al. 2013) validates the feasibility of fatigue growth measurements with DIC. Destrebecq et al. (2011) conducted the analysis for reinforced concrete beams using DIC, and the crack detection and measurement results showed that DIC provides high precision results for damage detection and load capacity evaluation. Dizaji (Dizaji et al. 2018) leveraged DIC for steel beam structural identification, and this research work has demonstrated that the DIC provides sufficient full-field surface deformation and offers a potential chance for full-filed structural identification, which is not possible for conventional discrete sensor response.

Damage prorogation, especially cracking, is also the primary damage index for existing concrete structures. Conventional strain gauge or vision-based sensing enable crack detection, but these methods are constrained by the measurement area and pixel resolution. Desai (2016) also demonstrated that the real-world early damage detection using DIC still requires more work to be conducted; however, early damage detection with small strain measurement has been achieved using the DIC method under controlled laboratory conditions. Researchers have used the 2D or 3D DIC method for small-scale laboratory specimen measurements with good accuracy, but it is much more difficult to conduct large or full-scale concrete structure measurements, including laboratory testing. The primary aspect to be considered is the balance between accuracy and view field, and the speckle pattern also must be carefully designed for rough surfaces.

In general, the early detection of cracks or other damages is useful for timely inspection and prevention. DIC provides sufficient surface information for damage detection, but interior damage propagation is another crucial concern. Acoustic emission (AE) provides accurate early detection feasibility for this scenario. It may provide more insight about the identification of different sources of damages. Debonding, microcracking and rupture damages are very difficult to monitor using ex- isting methods, and manual visual inspection is not completely reliable, which may result in many more subjective mistakes. AE is a common passive, nondestructive testing method which has been used for 50 years. It can provide inside information about the damage propagation within the structure. It uses a piezoelectric sensor to collect the elastic waves with a range of frequencies emitted by stressed or deformed materials, and the mechanical energy can be received and transferred with signal features, which can be used for damage detection and classification (Grosse et al. 2003). This method has been used for various damage detection studies in laboratory and field testing.

Sagar (Sagar and Prasad 2013) conducted the investigation of reinforced concrete beam cracking propagation, and the AE parameters such as frequency, duration, and amplitude were used to distinguish different cracks. He (Sagar and Rao 2015) also conducted flexural deformation experimentation to explore the cracking details and failure of concrete beams at different stages. Ohno (Ohno and Ohtsu 2010) developed the crack classification method from tensile mode to shear mode. An optimal proportion of the parameter analysis is provided for crack identification. Pohoryles (Pohoryles et al. 2017) demonstrated the use of the AE method to monitor the cracking and debonding processes of FRP retrofitted concrete beams, and the onset and development of debonding of FRP can be accurately determined during the experimental work. The AE method has also been used in field monitoring and testing. Nair (Nair and Cai 2010) discussed the available qualitative and quantitative techniques, and an assessment of the statistical quantitative analysis technique, intensity analysis, was also illustrated through two bridge health monitoring projects. Anay (Anay et al. 2015) also conducted on-site assessment of an old bridge without design plans: crack source and growth information was collected during the load testing, and intensity analysis showed that the significantly damaged region was located in the shear region with crack formation and growth.

The load capacity of existing structures decreases along with the material degradation, load conditions, etc., and early detection of damage is urgently required and useful in real applications, especially for old-aged structures. This paper aims to deploy a laboratory evaluation method for an old-aged reinforce concrete beam with limited structural information. This beam has served for more than 60 years, several types of damage can be observed from the surface, and there is a need to evaluate the actual mechanical state. To solve this problem, the AE method was used for early interior damage detection and determination of crack propagation, and the AE parameters were analyzed for damage detection. Furthermore, 3D-DIC was used to collect the detailed surface information, which can be processed for damage investigation, full-scale deformation measurement, and early damage detection.

This paper is organized as follows: a brief introduction 
about the experimental setup is introduced in section 2 . The corresponding experimental results are presented in section 3, including AE analysis and DIC measurement results. A brief discussion using these two methods is presented in section 4. Finally, the conclusion based on $\mathrm{AE}$ and DIC methods for old-aged beam investigation is presented in section 5 .

\section{Experimental setup}

\subsection{Description of the old-aged reinforced con- crete beam}

This reinforced concrete beam had been used for bearing cables in an electrical transformer substation. As part of the structure, it was exposed to a wet and warm environment for almost 60 years. It was removed from the structure due to the replacement of the substation, and then transported to the laboratory for investigation. Since the construction period was early, minimal structural information about this old-aged beam, including the concrete strength, reinforcement ratio, etc., exists.

The dimensional characteristics are as follows: the beam length is $7.86 \mathrm{~m}$, with a T-shaped cross section, and there are three diaphragms along the beam. The specific dimensions are shown in Fig. 1. There is significant visible damage on the surface of the beam: Fig. 2 shows 5 classic damage types, including exposed reinforcing steel, spalling, and cracks. An exposed reinforcing bar can be found on the top of the beam and few diagrams, while longitudinal cracks and network cracks can also be observed along the bottom of the beam and side surface. Spallings can also be observed on the top and sides of the beam. Both ends of the beam were slightly damaged because of the movement, so two cubic concrete attachments to the beam were produced for better support conditions. All of these surface damages indicate that this beam was terribly degraded, and the actual mechanism characteristics should be investigated by means of full-scale testing and measurement.

\subsection{Load setup}

The aim of this study is to determine the actual mechanical stage and damage propagation of this old-aged beam subjected to bending loads. The experiment adopted a four-point load test, and a $500 \mathrm{kN}$ hydraulic servo actuator was selected for load application. A steel I-beam was placed as a spreader beam beneath the actuator to transfer the concentrated force into two point force. A pin and triangle plate were selected to establish the simple support condition, and the specific installation can be observed in Fig. 1. Load was applied with incremental displacement of $2 \mathrm{~mm}$ after the first crack was observed. In order to determine the structural response using AE and DIC, 5 load cycles were conducted, controlled with incremental displacement, and then the load increment was used until the failure of the beam during

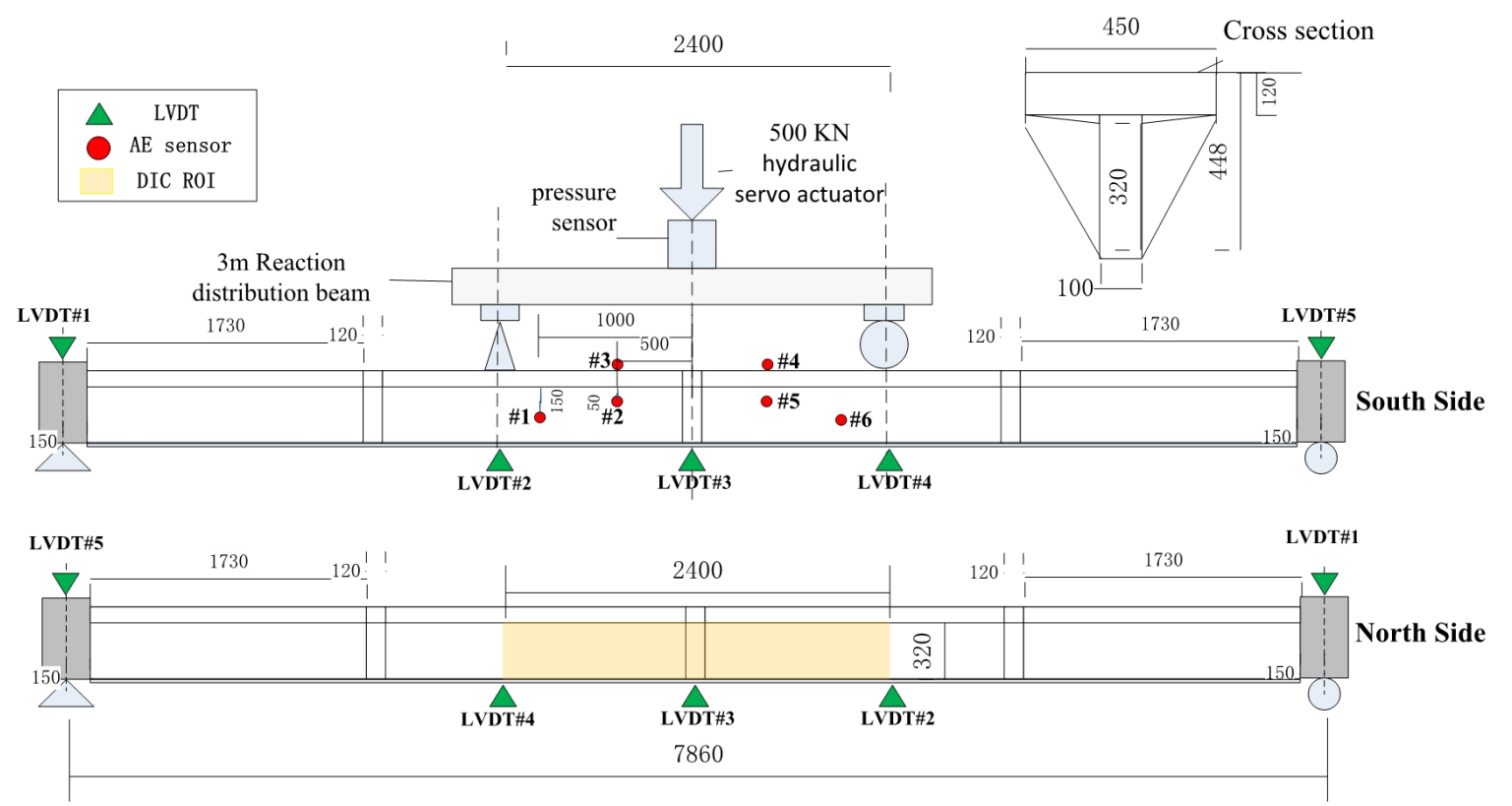

Fig. 1 Specific dimensions and sensor location.
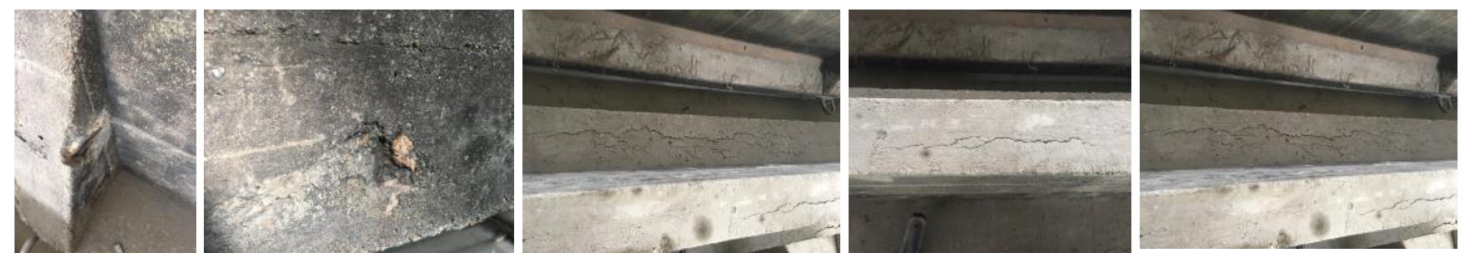

Fig. 2 Apparent existing damages on the concrete beam surface. 
the final load stage. Conventional discrete displacement sensors were installed at 5 locations: the center of the beam, right and left supports, and both sides of the two applied loading positions. The beam did not completely fail, and a larger load could not be applied due to the maximum distance limitations of the actuator. There were obvious damages at the end of the experiment. Specific results will be explained in the next section.

\subsection{Acoustic emission setup}

The AE data acquisition system used in this study is a 16 channel AEwin system, called the SENSOR HIGHWAY II AE system. Six PAC-broadband sensors with an upper frequency range of $1000 \mathrm{kHz}$ were fixed: 4 of them were installed at symmetrical positions on both sides of the middle span, and the other 2 sensors were installed atop the beam as shown in Figs. 1 and 3. The pre-amplifiers were selected with $40 \mathrm{~dB}$ AE signals to ensure a high signal-to-noise ratio. Based on the experimental environment noise, the threshold value in AEwin software was set as $35 \mathrm{~dB}$, and the sampling rate was set as 10 $\mathrm{MHz}$. Additionally, vacuum grease was used for coupling between the sensor and the surface of the beam. The application of the couplant can help to prevent noise transmission from surface roughness and air gaps. The advantage of $\mathrm{AE}$ is that the damage can be linked with AE signals, and even microcracks which are invisible to the human eye. These damages can be easily identified by AE parameters. Thus, it is important to collect accurate $\mathrm{AE}$ parameters, such as amplitude, average frequency, rise time, energy, etc., which are useful for damage identification in this study.

\subsection{The digital image correlation setup}

As mentioned above, conventional discrete sensors provide the limited response of the entire structure. They may be useful for small-scale beams in a laboratory: full-field measurement is not possible for full-scale structures. In this study, due to the large span of the beam, strain gauges and LVDT are cost and labor-consuming. DIC provides full-field measurements of both displacement and strain. The region of interest, denoted as ROI, is chosen in the reference image. In this study, ROI corresponds to a rectangle-shaped area of $2400 \times 320 \mathrm{~mm}$ which is located between the applied loading points and shown by a light orange area in Fig. 1. DANTEC Istra 4D software was selected for camera calibration and image evaluation. This software is effective for online calibration for multiple pairs of cameras, and it also provides reliable image stitching for 3 pairs of camera images in this study. The $2448 \times 2048$ pixel images were acquired with 8-bit CCD cameras. DIC requires a random distribution of speckles on all images. The first method is randomly painting the sizable black or gray spots, and another way is to print the random speckles of predetermined size (diameter of $1 \mathrm{~mm}$ ) with hydrographic transfer printing papers in advance, and then to transfer the speckles onto the surface of the beam. In this study, due to the long-term exposure of the rough surface, it is difficult to paint satisfactory speckles: a special hydrographic transfer printing method was chosen, and few painted speckles were used to recapitulate the small missing area.

The accuracy of DIC is based on the pixel resolution and size of the optical sensor, along with the distance between camera and specimen. A higher pixel resolution results in a finer representation of response (displacement), while the distance will be smaller with an attenuated measured ROI region (Dizaji et al. 2018). The physical size also represents more pixels, which can provide more robustness against noise and lighting conditions. The longer distance can result in a wider measured region, but the accuracy will decrease. There is a trade-off between camera cost, distance and measurement accuracy. The region of interest is the primary choice in the present study, and it is also not possible to measure the entire rectangular area with limited pairs of cameras. In this study, three pairs of cameras were selected: as shown in Fig. 4, each pair of cameras measures an area of $800 \times 320 \mathrm{~mm}^{2}$. Based on Equation (1) below, the measured width in this study is $320 \mathrm{~mm}$, and the image width is 2448 pixels. As Equation (1) shows, $\mathrm{R}$ denotes the physical size of each pixel, which is represented as $0.13 \mathrm{~mm} /$ pixel. Considering that the DANTEC

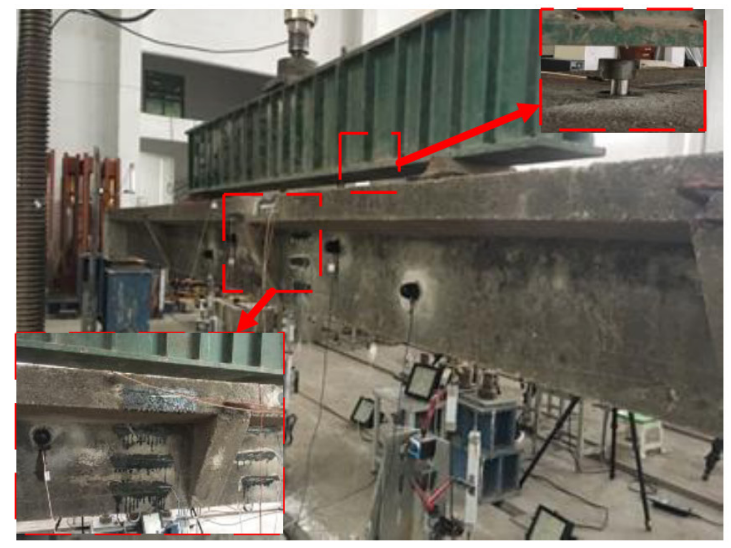

(a)Deployment of AE channels

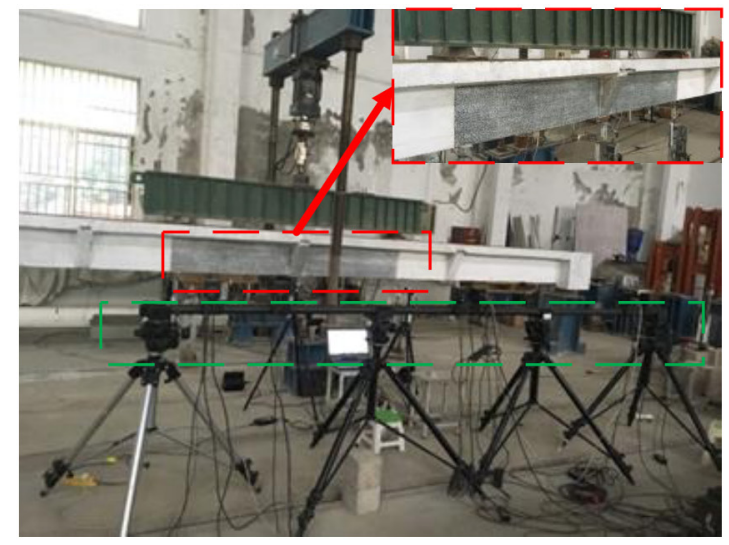

(b) Deployment of DIC

Fig. 3 Deployment of AE and DIC. 
software provides more than 0.1 pixel processing accuracy, the ideal accuracy of the measured displacement values in this study is less than $0.013 \mathrm{~mm}$.

$$
R(\mathrm{~mm} / \text { pixel })=\frac{\text { MeasuredWidth }(\mathrm{mm})}{\text { ImageWidth }(\text { pixel })}
$$

It is observed that the quality of the speckle pattern used in DIC directly affects the result accuracy. The structural surface must be covered with a random speckle pattern which is deformed by the object. It is important to assess the speckle pattern quality before the measurement. The gray level distribution of images can indicate the quality of the speckle pattern. Figure 5(a) shows the gray level distribution of the image with $2448 \times 2048$ pixels, and Fig. 5(b) shows the ROI region gray level distribution. All of these plots indicate that the random pattern is satisfactory, and according to reference (Pan et al. 2010), both types of numerical testing performed well.

Due to the large measurement area, three pairs of stereo-digital cameras were used. Istra $4 \mathrm{D}$ provides the image stitching module, in which the system can provide the full-field deformed information in uniform coordinates based on each of the local coordinates and calibration data. The calibration images were captured by each pair of cameras, and the calibration parameters, including intrinsic and extrinsic parameters, were computed. The reference images were set as the initial image before the load was applied. The sequence images were then uploaded, and start points were marked for feature matching and image stitching. In order to realize multiple image evaluation, a regular grid with equidistant grid points is laid over the image of the reference camera: each grid point defines the center of a squared image region in the reference image, and then the image correlation algorithm uses these regions to determine the 3D-position and tangential plane of the underlying object surface for each of the grid points. This square region is used to identify the corresponding area in another camera image.

\section{Experimental results and discussion}

\subsection{General description during three load stages}

As mentioned above, the first load stage was implemented by incremental displacement $(2 \mathrm{~mm}$ ) before the first crack was observed by visual inspection. As shown in Fig. 6(a), the first visible crack was completely observed near AE sensor 2, which appeared through the bottom and propagated up the side surface. The load was then decreased to zero for the incremental cycle load (load stage 2). In Fig. 7(a), there is an obvious decline at the value of $32.23 \mathrm{kN}$, and the corresponding load displacement is $13.74 \mathrm{~mm}$. As the load decreased to zero, there was also an obvious residual displacement, which indicates that the first new damage (crack) existed for this beam.

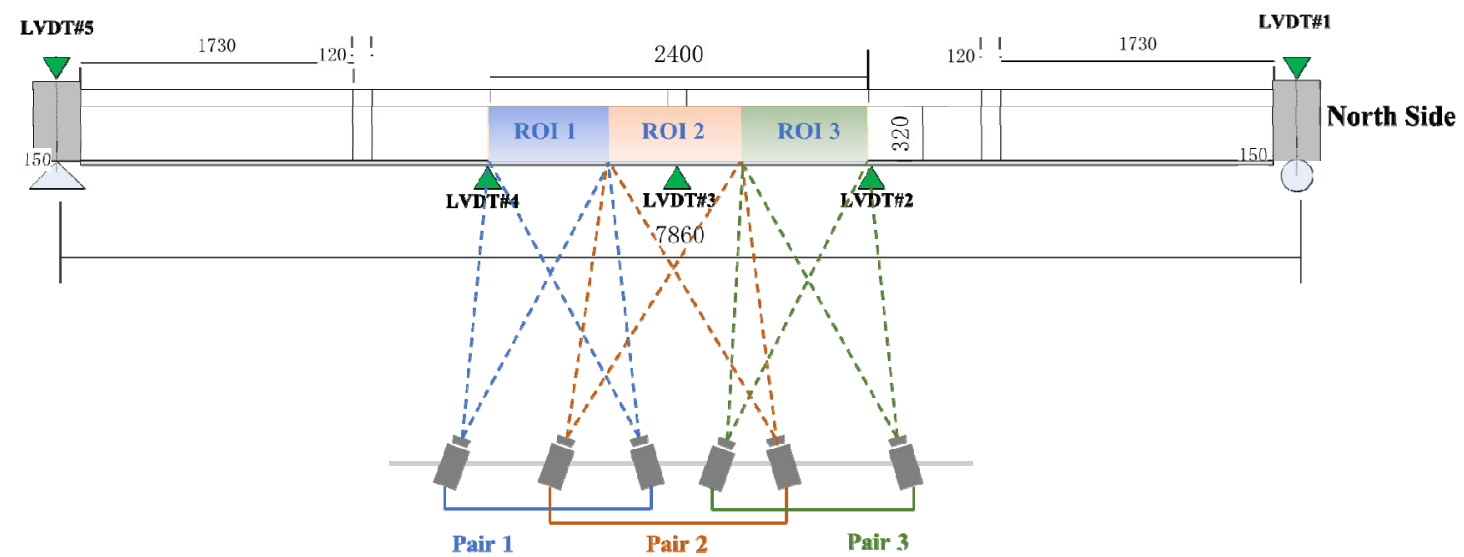

Fig. 4 Camera layout of DIC in the experiment.

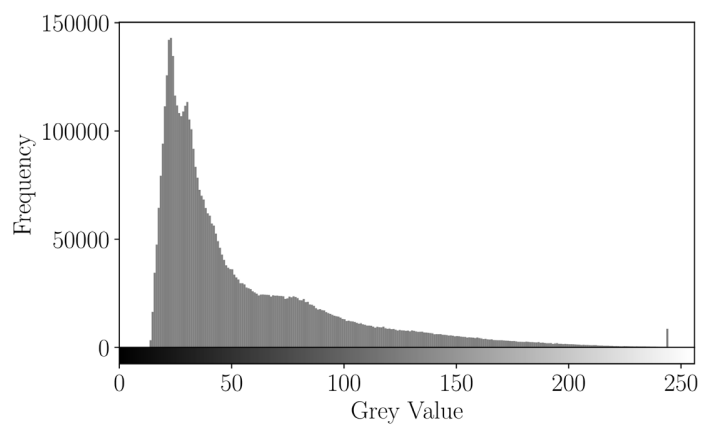

(a) Gray level of whole images

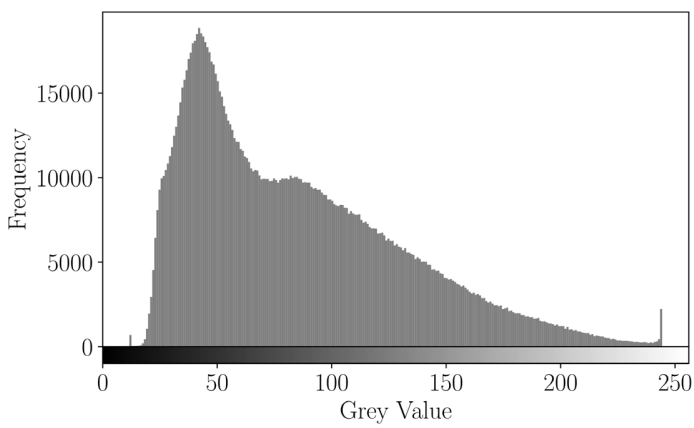

(b) ROI gray level of images

Fig. 5 Gray Level of Images. 
In load stage 2, the displacement control method with an increment of $2 \mathrm{~cm}$ was used for the increment cycle load. The specific load stage can be observed in Fig. 7(b). Despite the fact that the crack was formed and observed in the previous load stage, the displacement and load curve exhibited linearity before the load reached $32 \mathrm{kN}$ again; the load still rapidly decreased, and the beam began to enter the plastic mechanical stage shown in the last load cycle in Fig. 7(b).

After another two cracks were found, the load decreased to zero, and then the maximum load was applied to examine the failure mode and crack pattern of this old-aged beam. The displacement and load curve can be seen in Fig. 7(c): the load slowly increased to $44.28 \mathrm{kN}$, the displacement reached $184.37 \mathrm{~mm}$, and the vertical displacement of the middle span reached $187.78 \mathrm{~mm}$. Despite the fact that the vertical displacement is large enough for conventional design, the load can still be increased and exceed the actuator distance.

\subsection{Damage detection}

The AE parameters mentioned above have many features (Kocur and Vogel 2010; Shahidan et al. 2013). Several researchers use the amplitude, rise time, average frequency, count and energy parameters to indicate the damage occurrence and to determine the damage characteristics. Energy is one of the indexes for abnormal signal detection, which can easily identify the crack occurrence along with the amplitude value.

AE sensors 2 and 5 are close to the middle span, as Fig. 8 shows, and the energy plot exhibits the same trend. When the load reached the maximum value, there was a significant increase of the energy plot in channel 2, which indicates that the old-aged beam released the first crack stress wave. The energy parameter can be used as a good prediction index for crack detection. The cumula-

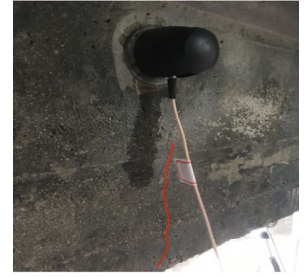

(a) 1 st observed crack near AE 2

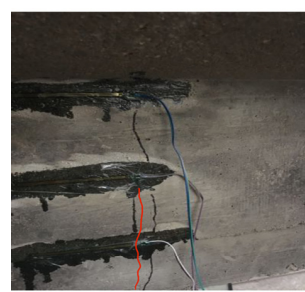

(b) 2nd observed crack in the middle Fig. 6 Observed early cracks.

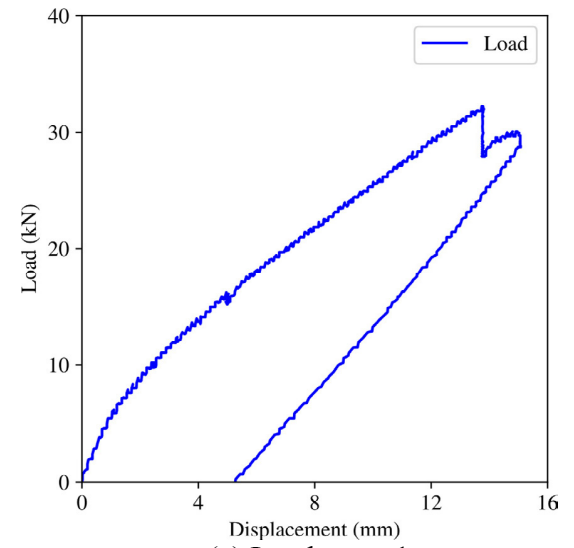

(a) Load stage 1

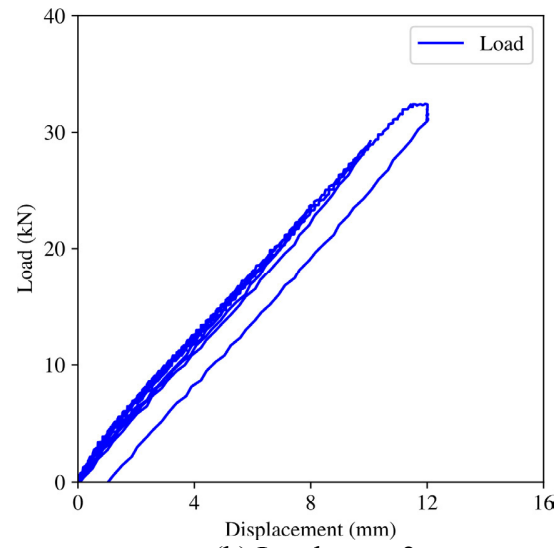

(b) Load stage 2

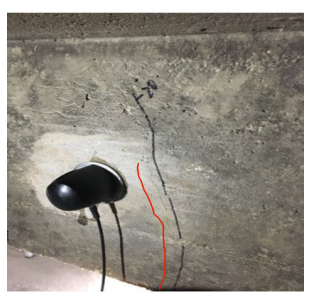

(c) 3rd observed crack near AE 5

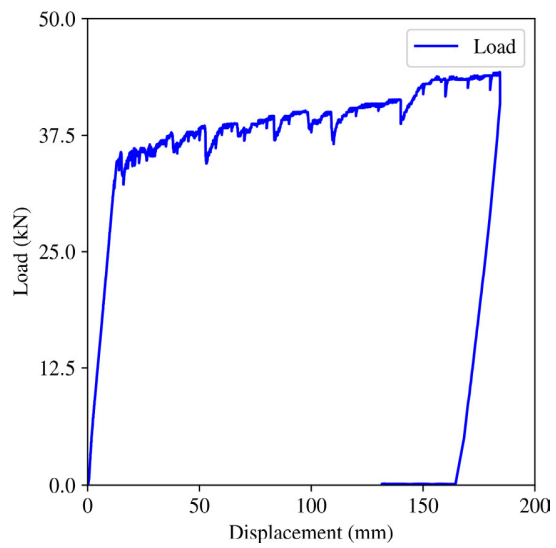

(c) Load stage 3

Fig. 7 Load and displacement plot in the three load stages.

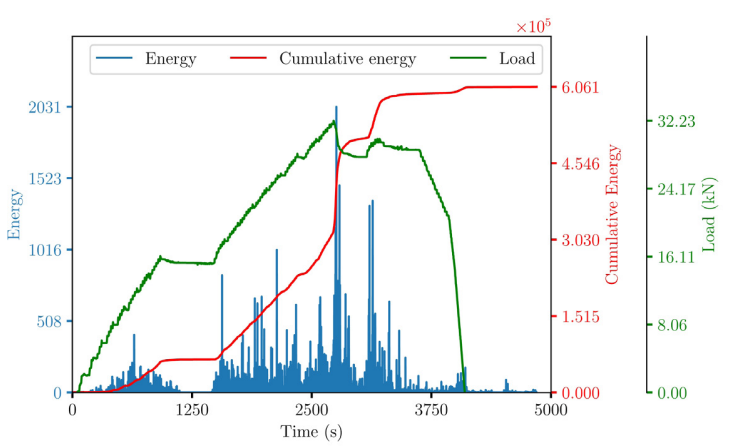

(a) Energy and cumulative plot in Channel 2

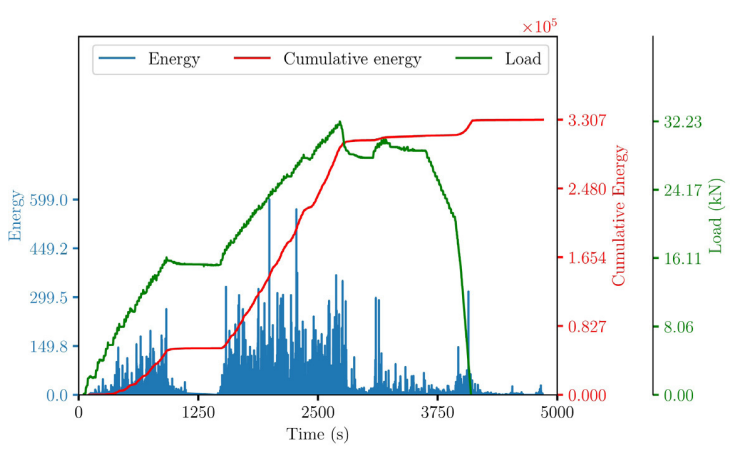

(b) Energy and cumulative plot in Channel 5

Fig. 8 Energy and cumulative plot in AE channels in load stage 1. 
tive value of energy is a better parameter to represent the overall state and integrity of the beam. As can be seen in Fig. 8, the energy parameter data exhibited the same trend as the load increased and decreased. There is a small range of signals at the beginning of the load, and there are few signals between the load processes, which is due to the vision operation during that process: this also can be observed on the displacement curve. As the load increased, the number of AE signals became larger and crowded, which means that there were numerous elastic stress activities inside the structure. There was a significant decrease of the peak of the load curve, and the energy showed great fluctuation during this process. The cumulative energy curve of channel 2 shows the largest fluctuation, especially at the maximum load; on the contrary, channel 5 , the symmetry point at the other side of the beam, shows less fluctuation. Since these two points were the closest locations near the middle span, it was concluded that a microcrack may have formed at channel 2: this was verified by the visible results.

The energy plot during the load cycle stage can be seen in Fig .9. The energy amplitude increased at each load cycle, and the cumulative energy plot increased when the load reached the maximum value in each cycle. Figure 9 shows that the AE activity around channel 2 still generated more hits, the parameter values increase with load magnitude, and the discontinuities in the concrete beam do not expand until the former stress is exceeded, which is known as the Kaiser effect; the acoustic events do not occur at the initial load stage in each cycle, and they began to occur when the load exceeded the last load value. This phenomenon can be clearly observed in each parameter plot in Fig. 9. The number of hits significantly increases, the amplitudes of the hits reach the highest values between 30 to $32 \mathrm{kN}$, the cumulative curve exhibits a more clear signal trend, and there exist 3 significantly high rates of increase when the load reaches 30 $\mathrm{kN}$ in each cycle. This matches the visible result that 1 existing crack propagated and 2 new cracks appeared, as observed in Figs. 6(b) and 6(c).

The energy plot during the final load stage can be seen in Fig. 10. As the cumulative energy curve shows, the beam still generated plenty of stress waves. In contrast with other research (Fotouhi et al. 2016), the energy parameter will decrease after the load is decreased, and the cumulative energy curve will plateau. In this present study, continuously increasing energy and a cumulative plot show that the crack still propagated, and the debonding between concrete and steel also occurred simultaneously. Contrary to the previous signal parameter trend in which a high rate of increase showed that a new crack appeared, it is difficult to clarify the new crack signal due to the dense plot, but the cumulative plot of the energy signal also indicates the crack movement near each AE sensor position. In fact, the final crack pattern has been formed in this load stage, but each crack still propagated with the increasing load. Since the magnitude of each channel is nearly identical, there exist several rapid increases in each channel. For channel 2, as Fig. 10(a) shows, there are two rapid increases at $5000 \mathrm{~s}$ and $8000 \mathrm{~s}$, while channel 5 exhibited 1 increase at $6000 \mathrm{~s}$. The increasing and decreasing load fluctuation trends

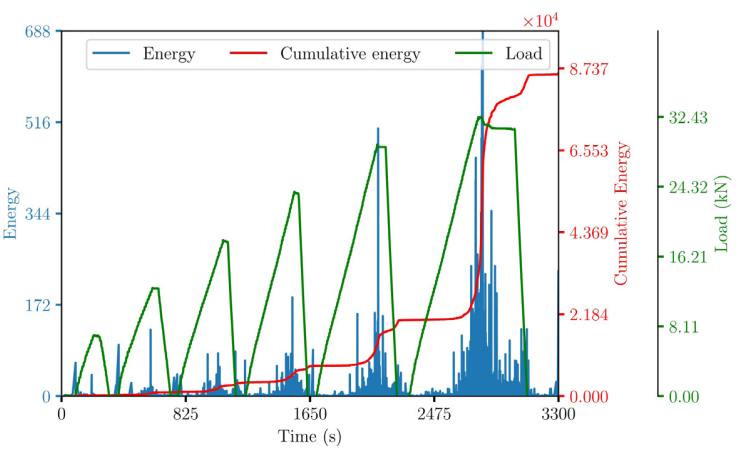

(a) Energy and cumulative plot in Channel 2

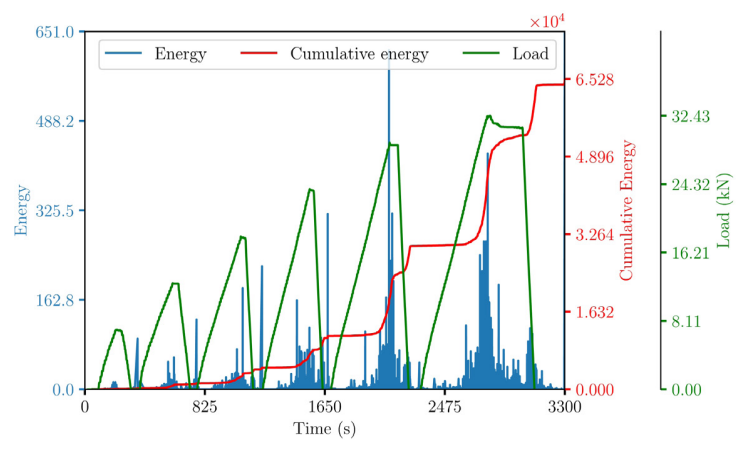

(b) Energy and cumulative plot in Channel 5

Fig. 9 Energy and cumulative plot in AE channels in load stage 2.

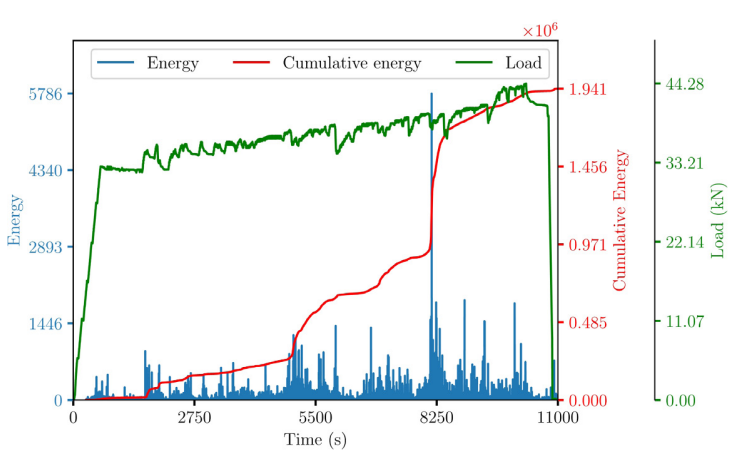

(a) Energy and cumulative plot in Channel 2

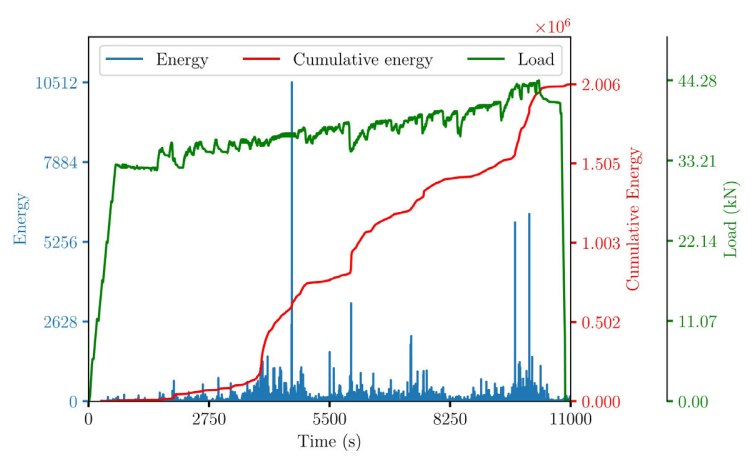

(b) Energy and cumulative plot in Channel 5

Fig. 10 Energy and cumulative plot in AE channels in load stage 3. 
usually indicate the debonding between concrete and steel along the entire beam.

At the same time, DIC can provide the full-field strain result for crack pattern recognition. Despite the lighting conditions and camera field of the center region lacking results for the middle span, the distribution contour plot can still provide information about crack positioning and movement. The DIC strain contour plot indicates the crack distribution and pattern seen in Fig. 11(a) as the load increased to $6 \mathrm{kN}$ : despite the missing region in the center of the middle span, 4 crack outlines appeared in each side, whereas it is not possible to discover each crack pattern by visual observation.

When the load increased to $28.31 \mathrm{kN}$, negative strain developed around the right side of Fig. 11(b), which indicates the redistribution of stress in this region. A microscopic crack may have appeared. This matched the experimental and $\mathrm{AE}$ results mentioned in the previous section, and the right side in DIC images refers to the left side in the AE result seen in Fig .1.
As the load increased, a high-level strain field developed on the left side, and strain redistribution also occurred near this region. Fig. 12(a) can clearly demonstrate two cracks on each side. More cracks and their movement alongside the surface can be detected based on each of the plots in Figs. 12(b)-(d). The concrete surface and attached speckle pattern started to spall, and several margins exist on the plot. Comparing the final plot of Fig. 12(d) with the Fig. 12(a) plot, when the crack pattern can be first recognized, the crack patterns, positions and their movements with the increasing load can be clearly matched. These plots perfectly indicated the crack patterns and their positions, and the specific propagation of each crack can also be detected based on the strain field results. However, the strain value around each crack may be invalid due to the stress redistribution and noise effect.

On account of the concrete bending capacity, the surface strain distribution is relatively smaller than those of steel or other high elasticity modulus materials, and the

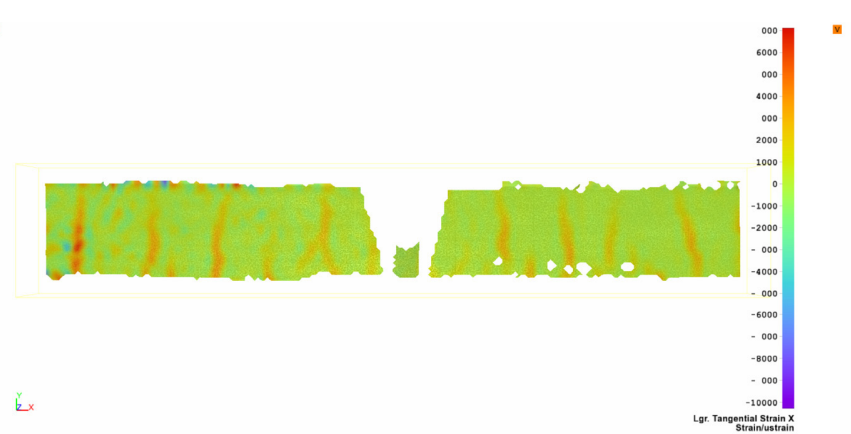

(a) Strain distribution at $6 \mathrm{kN}$

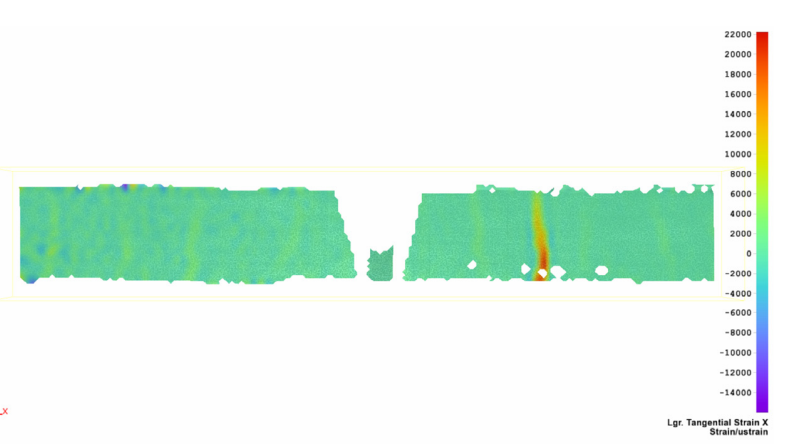

(b) Strain distribution at $28.31 \mathrm{kN}$

Fig. 11 DIC strain field results in load stages 1 and 2.

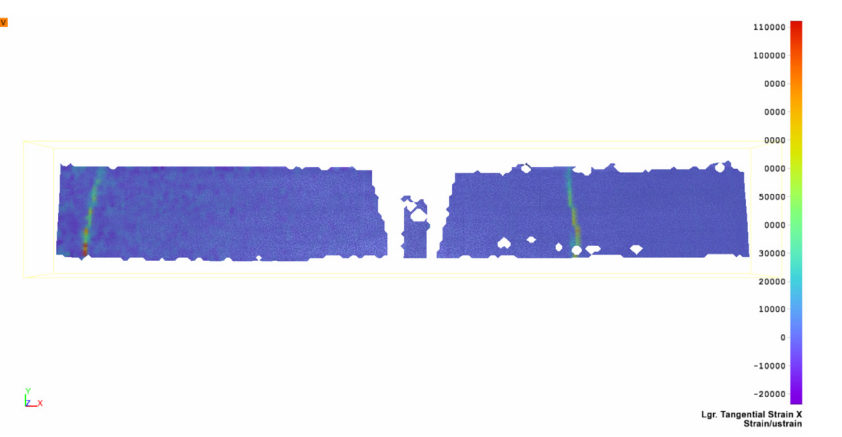

(a) Strain distribution at $36.09 \mathrm{kN}$

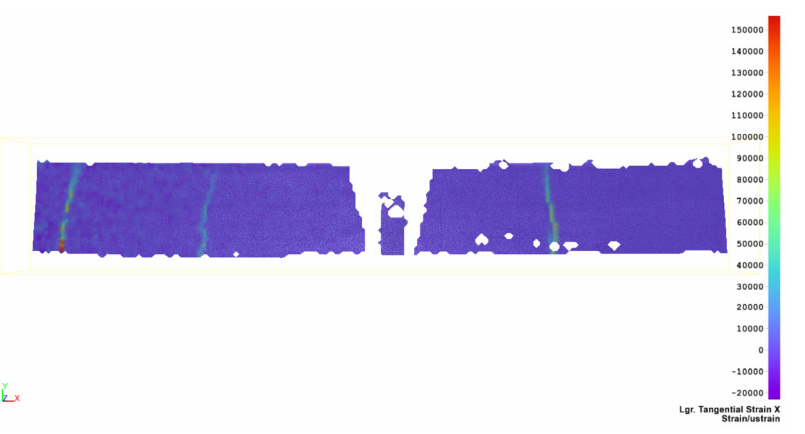

(b) Strain distribution at $36.11 \mathrm{kN}$

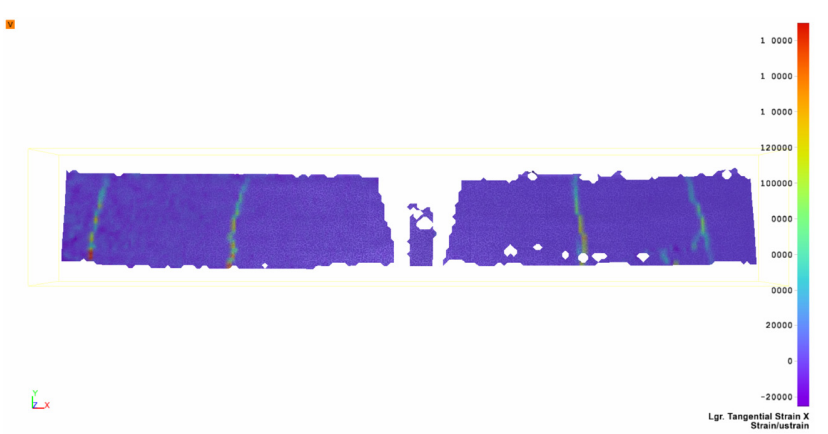

(c) Strain distribution at $37.84 \mathrm{kN}$

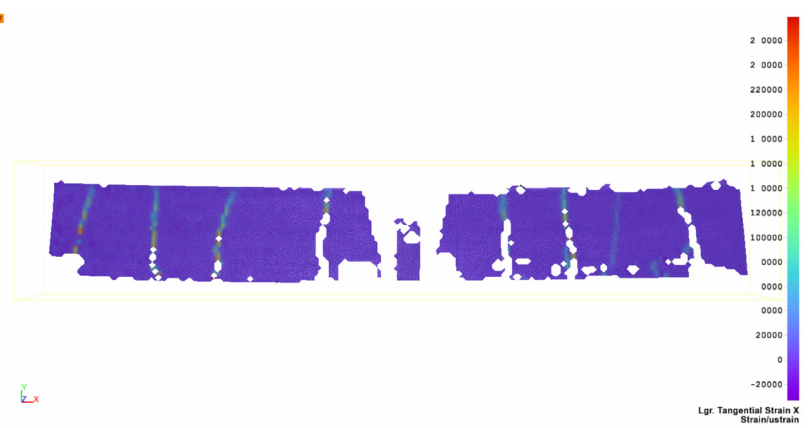

(d) Strain distribution at $38.71 \mathrm{kN}$

Fig. 12 DIC strain field results in load stage 3. 
strain field result before the load, which is also recognized as noise level, can affect the strain accuracy in DIC. The noise level of strain in general is between 50 to 150 $\mu \in$. This test also cannot reach a satisfactory strain field result in each step using Istra $4 \mathrm{D}$. This situation may be caused by the lighting conditions, few broken speckles and image resolution. Because the noise level can overwhelm true strain values on the structure surface, the specific strain value in each grid of the DIC results may not indicate the real strain, but the comprehensive strain distribution and movement can ideally indicate the surface deformation information, which can be used for early damage detection.

\subsection{Damage evaluation}

Damage evaluation can be achieved by the relationship between RA and AF, and b-value analysis also provides clear information about the structural mechanical state. Furthermore, the deflection measurement results from DIC also provide information about specific crack movement and overall deformation of the structure.

Referring to (Aggelis 2011), Average Frequency against RA value is the classic method to identify the crack characteristics and movement of tensile cracks and shear cracks. RA means Rise time divided by Amplitude, which is measured in $\mu s / V, m s / V$ or $s / V$ in Equation (2), and AF means Counts divided by durable time, which is measured in $\mathrm{kHz}$ as shown in Equation (3). The unit of rise time and durable time in this present study is $\mu s$. The amplitude is $\mathrm{dB}$, which can be converted into $\mu s / V$ by Equation (4). The tensile crack exhibits low rise time and high frequency, while shear movement and failure stage exhibit high rise time and low frequency (Shahidan et al. 2013).

$$
\begin{aligned}
& R A=\frac{\text { Risetime }(R T)}{\text { Amplitude }} \\
& \text { AverageFrequency }=\frac{\text { AEcounts }}{\text { Durationtime }} \\
& d B=20 \log \left(V_{p}\right)
\end{aligned}
$$

It has been proposed (Omondi et al. 2016) that ratios of 10 to 50 for the abscissa can clearly demonstrate the types of damage, including tensile and shear. For the present study, the ratio of 50 was selected to show the shift process during the load stage based on the hit characteristics. Averaging AE hits of 50 blocks was chosen due to the numerous recorded hits.

Figure 13 shows the AF against RA plots for three load stages in this study. The black line is shown to clarify the tensile and shear cracking, and the mean value of each cluster is shown in blue points. The first load stage shows few hits, and most of them are located in the left region (tensile). Fewer hits were found in the cycle load stage, but the distribution of these hits is still located in the same region, despite the cracks which were found. Quite a large number of hits were found in load stage 3, and the center moved towards the right side; cracks were visibly observed along the beam, and other shear-related mechanisms occurred in this stage. There is an obvious shift trend of hits from tensile to shear regions. However, the majority of the hits are still located in the tensile region, and the mean value of these three plots still locates in the tensile region. This result matches the bending capacity investigation of this study.

The differences of cracks can be linked with the degrees of structures. Microcracks generate more hits with lower amplitude, while macrocracks generate fewer hits, but higher amplitude. When a crack propagates, most of the energy is released with numerous hits of small amplitude (Colombo et al. 2003). The b-value is the index to take these factors into account for damage evaluation. It can be calculated as:

$$
\log _{10} N=a-b \frac{A_{d B}}{20}
$$

where $N$ denotes the incremental frequency of the AE hits with amplitude greater than the threshold, $A_{d B}$ is the peak amplitude of $\mathrm{AE}$ events in $\mathrm{dB},{ }^{\prime} a$ ' is the empirical constant, and ' $b$ ' is the b-value. This shows the fact that the b-value is high for distributed microcracks and low for macrocrack occurrence.

Figure 14 shows the b-values during all load stages,

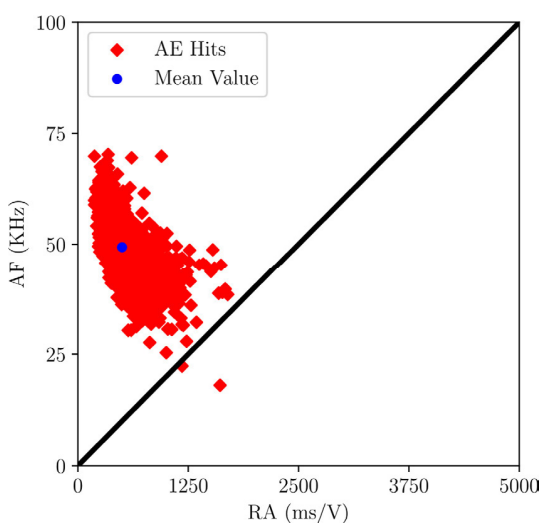

(a) RA/AF in load stage 1

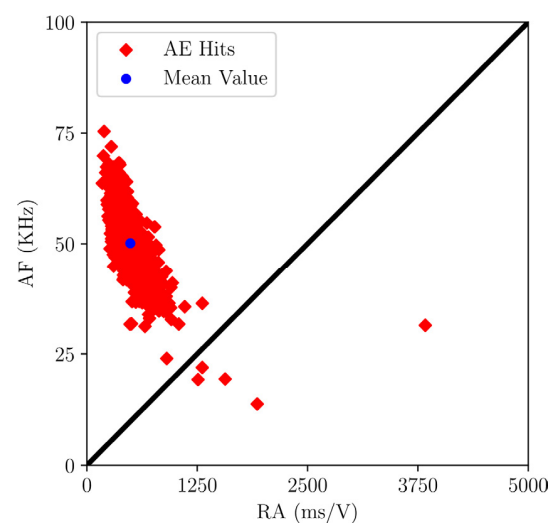

(b) RA/AF in load stage 2

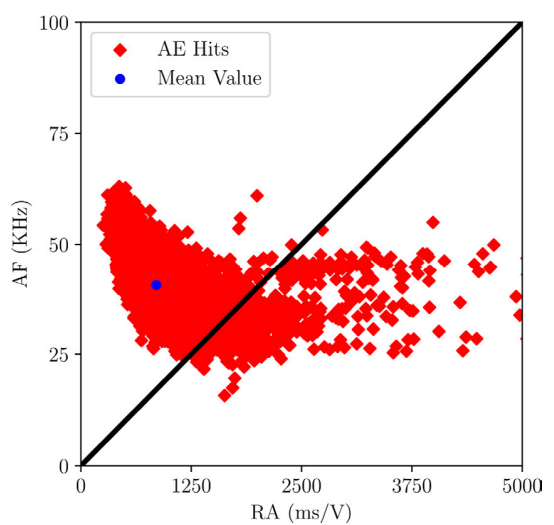

(c) RA/AF in load stage 3

Fig. 13 Plot of AF against RA value for channel 2 in three load stages. 
Table 1 Crack location and mean spacing values $(\mathrm{mm})$.

\begin{tabular}{ccccccccc}
\hline Crack number & Crack 1 & Crack 2 & Crack 3 & Crack 4 & Crack 5 & Crack 6 & Crack 7 & Crack 8 \\
\hline $\begin{array}{c}\text { Location } \\
\text { Mean width }\end{array}$ & 55.1 & 345.2 & 556.5 & 890.4 & 1547.7 & 1760.1 & 1916.7 & 2207.0 \\
\hline
\end{tabular}

and the load versus time plot is also provided for clear comparison. The least square fitting was adopted to obtain the negative slope of the line, which is the b-value. The cumulative number of AE hits in steps of $5 \mathrm{~dB}$ and the group of 100 hits were chosen to show the b-value trend. It can be observed in Fig. 14 that b-values are more clustered before the load reaches its maximum. There is a significant decrease of $b$-value when the load reaches the maximum. The minimum $b$-value in each load stage matches the crack opening and moving process, and the slippage of rebar also indicates the higher b-value shown for the last load stage. The b-value decrease corresponds to the phase of the load test when the crack is opening, providing a good representation of microscale and macroscale damage evolution on the beam.

The distribution of the longitudinal displacement in the tensile zone exerts a significant effect on the cracking process, and DIC provides good resolution of this displacement measurement. The cracking process and the opening location of cracks during the test can be clearly identified.

Figure 15 shows an example of crack detection based on longitudinal displacement distribution at a distance of $8 \mathrm{~cm}$ from the bottom for four loading levels. It can be observed that each discontinuity represents the existence of a crack, and the location can be calculated by the abscissa: the vertical amplitude of each discontinuity corresponds to the crack opening and specific width. There exist 8 cracks in Fig. 15, and the crack spacing can be obtained by the difference between relevant crack locations. Owing to the high resolution of DIC, the crack opening location and average spacing can be calculated in a low-cost and safe manner. The spacing of each crack can be calculated, and the results are shown in Table 1.

As mentioned above, the actual crack widths correspond to the amplitude of the discontinuities. Figure 16 provides the crack width propagation in each load stage. It is obvious that each crack behaves in a different way, and larger values are observed for crack 6 . There is a difference for crack 6 with respect to cycle load, in that the initial crack width is much larger than others, and this may be caused by the interlocking effects which prevent existing cracks from closing. Finally, DIC proves that it is suitable for early measurement of crack opening and width movement, even from an uncracked state.

In addition to the longitudinal displacement measurement, the vertical field displacement results also provide sufficient information about the deflection and

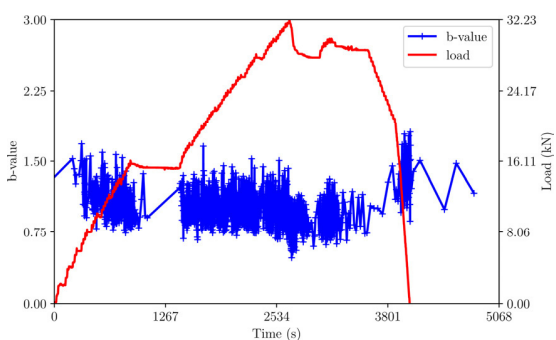

(a) b-value in load stage 1

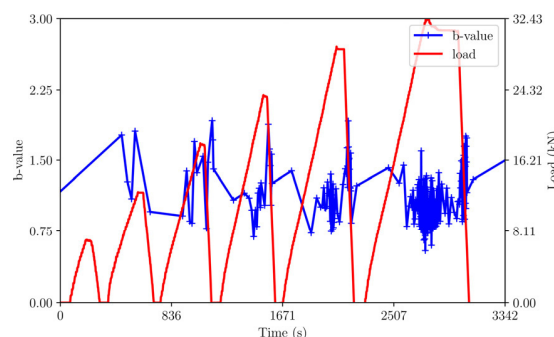

(b) b-value in load stage 2

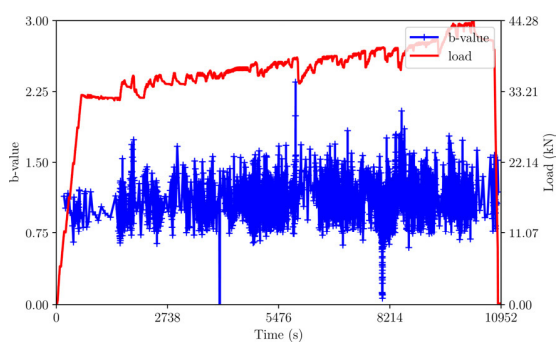

(c) b-value in load stage 3

Fig. 14 b-values of channel 2 in three load stages.

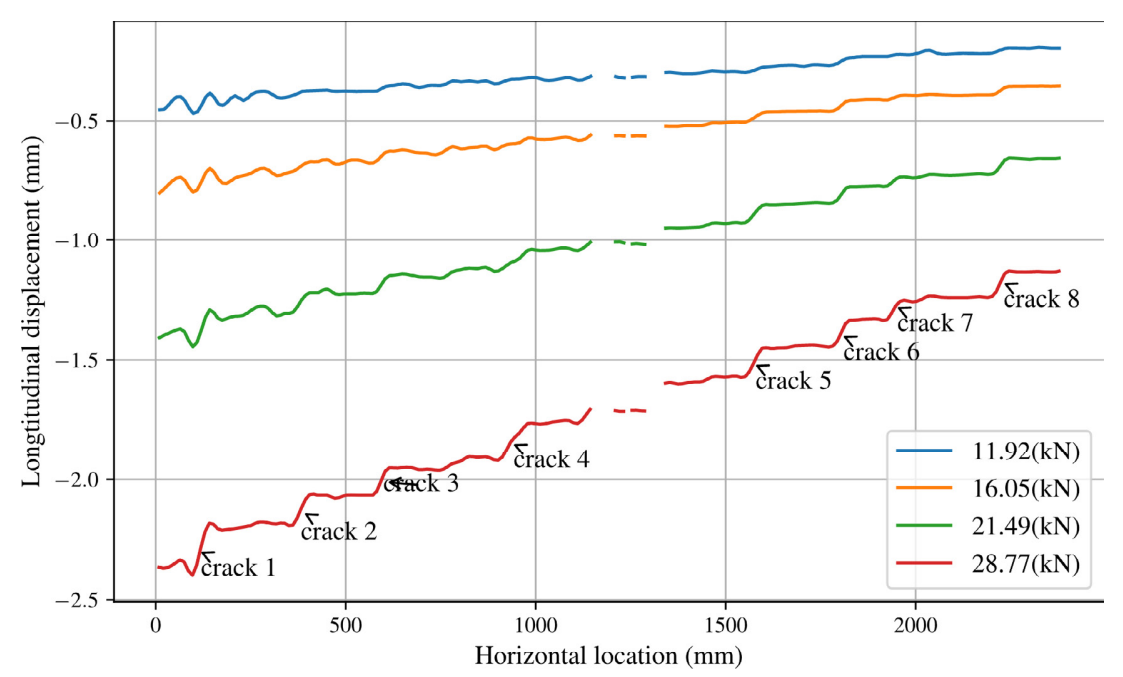

Fig. 15 Crack detection. 
curvature of this beam. Figures 17(a)-17(c) show the corresponding result before the load was applied: the vertical displacement remained uniformly less than 0.1 $\mathrm{mm}$, while the maximum fluctuation value was less than $0.03 \mathrm{~mm}$. DIC was used in discrete time in load stages 1 and 2, and was also used with a continuous acquired image in load stage 3 . It can be found that DIC captured the motion of ROI with perfect accuracy, and the red point in the plot matched the LVDT displacement value in each load stage. During the incremental load cycle, the DIC also measured the maximum and minimum displacement values in each cycle. Due to the device measurement sampling adjustment, there was an empty measurement in the red plot shown in Fig. 17(c), but the existing plot also provides the same trend of LVDT, since the deformation exceeds the field of view in each camera; the existing lighting reflection has effected the DIC image processing, and the measurement images of DIC cannot be processed at the end of this load stage.

From the knowledge of the vertical displacement field, it is possible to derive the flexural curvature during the entire load stage. In order to minimize the crack effects, the mean displacement values of the vertical line between each crack are selected. These values are fitted by a second degree polynomial, and the curvature is equal to twice the highest degree coefficient value. Figure 18 shows the curvature trend during the entire load stage. It is observed that the evolution of flexural curvature is linear in load stage 1 , and there exists residual curvature in load stage 2 as compared with the previous stage. The curvature is significantly increasing in load stage 3, while the load exhibits slow growth. This trend corresponds to the load displacement curve mentioned above, and the DIC measurement matches the deflection well during the entire load stage.

\section{Summary of observations}

Combined with $\mathrm{AE}$ and DIC processing results, it is shown that both methods provide good performance for early damage detection.

Compared with conventional methods, AE presents the advantage of ease of installation, and there is no risk of destroying the surface of the structure. Few AE sensors can monitor the complete structure activity. Moreover, it is reliable for early damage detection based on the AE parameters and their cumulative plots, such as energy. Early damage, mainly cracking, can be easily identified using magnitude or cumulative curve slope in this study, and the approximate damage position can also be located based on the different amplitude values of each of the AE sensor channels, cracks can be found near the AE sensor

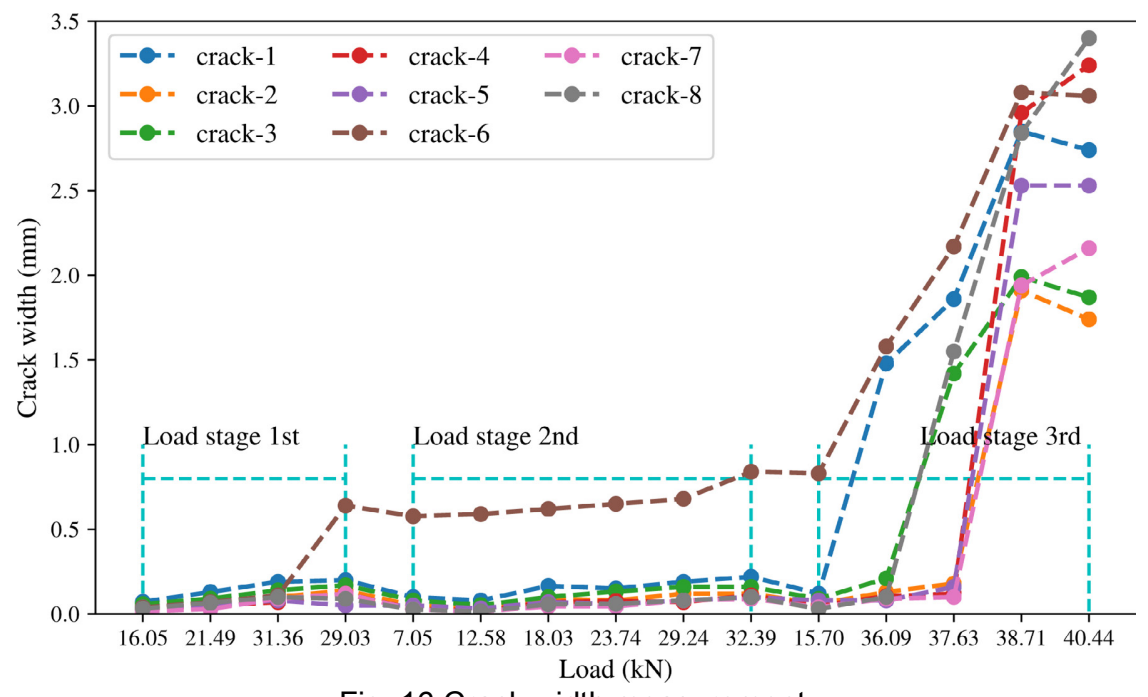

Fig. 16 Crack width measurement.

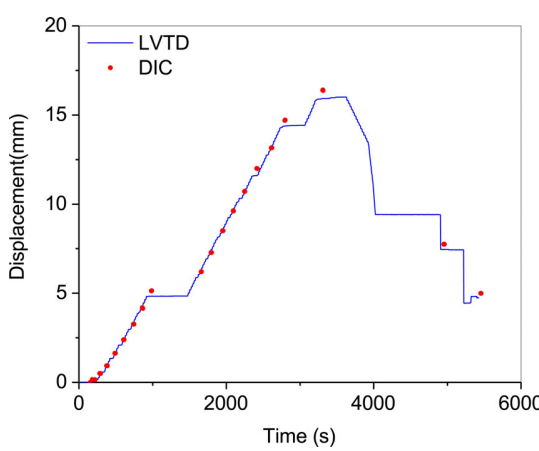

(a) Load stage 1

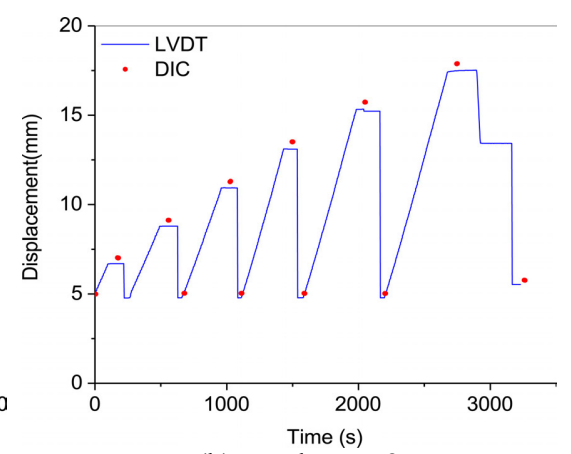

(b) Load stage 2

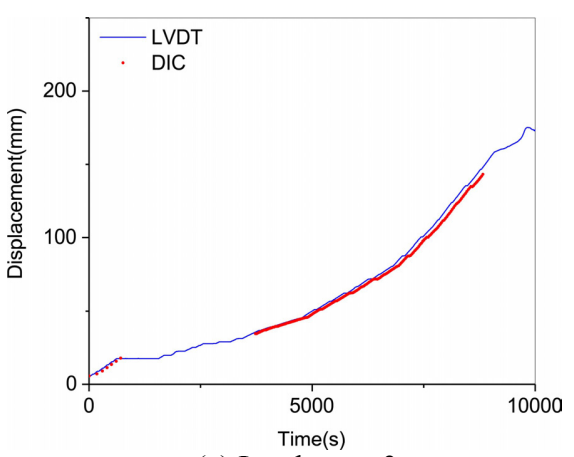

(c) Load stage 3

Fig. 17 Vertical displacement comparison between DIC and LVDT of midspan in three stages. 


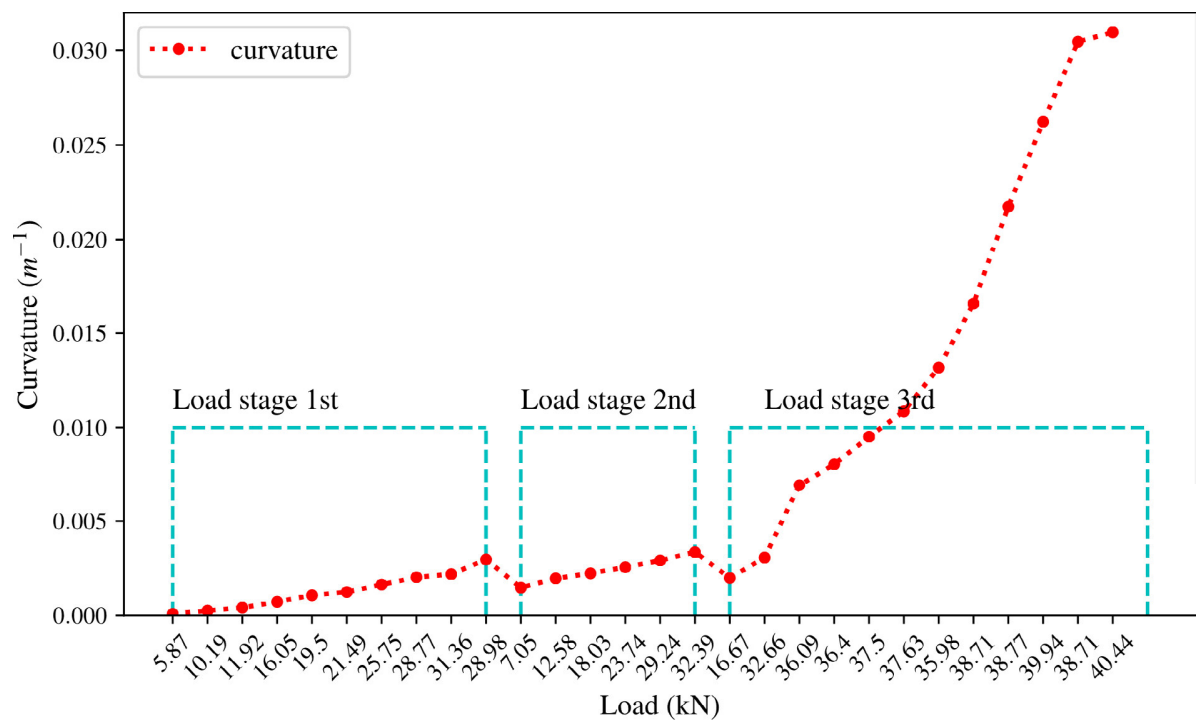

Fig. 18 DIC curvature measurement in whole load stage.

with biggest magnitude. At the end of the experiment, the debonding can also be identified. Due to the complex signal, it is difficult to clarify the new crack and existing crack propagation signs when the structure enters the plastic state, and more de-mixing work and classification work must be completed.

DIC also offers the advantage of full-field measurement without affecting the surface, and it provides large and accurate displacement measurement that conventional discrete sensors cannot realize in a cost-effective manner. However, the random speckles and light conditions should be carefully prepared before the experiment, which is labor-intensive work. For the large-scale measurement, more pairs of camera setup must be prepared with satisfactory accuracy. Though the specific strain result is not acceptable in this study, the strain field distribution serves as a good reference for crack pattern and propagation: the first crack and final crack position can be easily determined at the initial load stage, and this may serve as a good indicator for early damage detection. Moreover, DIC results are also restricted by image processing accuracy, missing areas in DIC plots may appear due to the image quality, and there is a balance between image integrity and measurement reliability, such that some important early damage information may be lost. DIC plots missed the middle span crack in this study. On the contrary, the AE parameters may provide relatively more accurate results during the initial load stage.

In general, these two methods can be integrated to handle early damage detection. AE provides more information about early damage occurrence time and location, and also provides the mechanical state evaluation based on the interior structural information. In addition, DIC provides the exterior surface full-field deformed information from global to local aspects, and the crack pattern prediction and full-field displacement measurement can also serve as good indicators for early damage detection and evaluation.

\section{Conclusion}

This paper highlights that AE and DIC are suitable for early damage detection for existing concrete structures, even without sufficient design information. A four-point bending experiment was conducted with combined $\mathrm{AE}$ and DIC methods. AE parameters were analyzed based on the experimental observations, and early damage occurrence and interior mechanical state can be identified with AE parameters. The damage propagation process can also be presented with RA vs. AF plot and b-value analysis. Displacement measurement was compared between LVDT and DIC, and the latter method supplies quantitative information about full-field structural deformation on the ROI surface from global and local aspects. Based on the strain full-field results in each load stage, the crack pattern can be precisely determined before the visible crack can be found. Overall, it can be concluded that the implementation of $\mathrm{AE}$ and DIC for early damage detection of full-scale concrete structure is feasible: these two methods, from interior and exterior aspects, respectively, prove useful in early damage detection. Further research must continue to focus on more accurate damage source location using AE signal data, and a more robust DIC measurement setup and processing method are also needed for full-scale structural measurement.

\section{Acknowledgements}

The authors greatly appreciate the financial support from the National Natural Science Foundation of China (Grant No. 51525801) and the Postgraduate Research \& Practice Innovation Program of Jiangsu Province, China (Project No. KYCX17_0120).

\section{References}

Agdas, D., Rice, J. A., Martinez, J. R. and Lasa, I. R., (2015). "Comparison of visual inspection and structural-health monitoring as bridge condition 
assessment methods." Journal of Performance of Constructed Facilities, 30(3), 04015049.

Aggelis, D. G., (2011). "Classification of cracking mode in concrete by acoustic emission parameters." Mechanics Research Communications, 38(3), 153-157.

Aktan, A. E., Catbas, F. N., Grimmelsman, K. A. and Tsikos, C. J., (2000). "Issues in infrastructure health monitoring for management." Journal of Engineering Mechanics, 126(7), 711-724.

Alipour, M., Washlesky, S. J. and Harris, D. K., (2019). "Field deployment and laboratory evaluation of 2D digital image correlation for deflection sensing in complex environments." Journal of Bridge Engineering, 24(4), 04019010.

Anay, R., Cortez, T. M., Jáuregui, D. V., ElBatanouny, M. K. and Ziehl, P., (2015). "On-site acoustic-emission monitoring for assessment of a prestressed concrete double-tee-beam bridge without plans." Journal of Performance of Constructed Facilities, 30(4), 04015062.

Carroll, J. D., Abuzaid, W., Lambros, J. and Sehitoglu, H., (2013). "High resolution digital image correlation measurements of strain accumulation in fatigue crack growth." International Journal of Fatigue, 57, 140-150.

Colombo, I. S., Main, I. G. and Forde, M. C., (2003). "Assessing damage of reinforced concrete beam using "b-value" analysis of acoustic emission signals." Journal of materials in civil engineering, 15(3), 280-286.

Desai, N., (2016). "Small-strain measurement in bridge connections using the digital image correlation (DIC) technique." In: Proc. Health Monitoring of Structural and Biological Systems, 2016, 980530.

Destrebecq, J.-F., Toussaint, E. and Ferrier, E., (2011). "Analysis of cracks and deformations in a full scale reinforced concrete beam using a digital image correlation technique." Experimental Mechanics, 51(6), 879-890.

Dizaji, M. S., Alipour, M. and Harris, D. K., (2018). "Leveraging full-field measurement from 3D digital image correlation for structural identification." Experimental Mechanics, 58(7), 1049-1066.

Farrar, C. R. and Lieven, N. A. J., (2006). "Damage prognosis: the future of structural health monitoring." Philosophical Transactions of the Royal Society A: Mathematical, Physical and Engineering Sciences, 365(1851), 623-632.

Feng, D. and Feng, M. Q., (2017). "Experimental validation of cost-effective vision-based structural health monitoring." Mechanical Systems and Signal Processing, 88, 199-211.

Fotouhi, M., Suwarta, P., Jalalvand, M., Czel, G. and Wisnom, M. R., (2016). "Detection of fibre fracture and ply fragmentation in thin-ply UD carbon/glass hybrid laminates using acoustic emission." Composites Part A: Applied Science and Manufacturing, 86, 66-76.

Grosse, C. U., Reinhardt, H. W. and Finck, F., (2003). "Signal-based acoustic emission techniques in civil engineering." Journal of materials in civil engineering, 15(3), 274-279.

Hou, S. and Wu, G., (2019)., "A low-cost IoT-based wireless sensor system for bridge displacement monitoring." Smart Materials and Structures, 28(8), 085047.

Hoult, N. A., Take, W. A., Lee, C. and Dutton, M., (2013). "Experimental accuracy of two dimensional strain measurements using digital image correlation." Engineering Structures, 46, 718-726.

Im, S. B., Hurlebaus, S. and Kang, Y. J., (2011). "Summary review of GPS technology for structural health monitoring." Journal of Structural Engineering, 139(10), 1653-1664.

Khuc, T. and Catbas, F. N., (2017). "Completely contactless structural health monitoring of real-life structures using cameras and computer vision." Structural Control and Health Monitoring, 24(1), e1852.

Ko, J. M. and Ni, Y. Q., (2005). "Technology developments in structural health monitoring of large-scale bridges." Engineering structures, 27(12), 1715-1725.

Kocur, G. K. and Vogel, T., (2010). "Classification of the damage condition of preloaded reinforced concrete slabs using parameter-based acoustic emission analysis." Construction and Building Materials, 24(12), 2332-2338.

Nair, A. and Cai, C. S., (2010). "Acoustic emission monitoring of bridges: Review and case studies." Engineering structures, 32(6), 1704-1714.

Ohno, K. and Ohtsu, M., (2010). "Crack classification in concrete based on acoustic emission." Construction and Building Materials, 24(12), 2339-2346.

Omondi, B., Aggelis, D. G., Sol, H. and Sitters, C., (2016). "Improved crack monitoring in structural concrete by combined acoustic emission and digital image correlation techniques." Structural Health Monitoring, 15(3), 359-378.

$\mathrm{Ou}, \mathrm{J}$. and Li, H., (2010). "Structural health monitoring in mainland China: review and future trends." Structural health monitoring, 9(3), 219-231.

Pan, B., Lu, Z. and Xie, H., (2010). "Mean intensity gradient: an effective global parameter for quality assessment of the speckle patterns used in digital image correlation." Optics and Lasers in Engineering, 48(4), 469-477.

Park, J.-W., Lee, J.-J., Jung, H.-J. and Myung, H., (2010). "Vision-based displacement measurement method for high-rise building structures using partitioning approach." Ndt \& E International, 43(7), 642-647.

Pohoryles, D. A., Melo, J., Rossetto, T., Fabian, M., McCague, C., Stavrianaki, K., Lishman, B. and Sargeant, B., (2017). "Use of DIC and AE for monitoring effective strain and debonding in FRP and FRCM-retrofitted RC beams." Journal of Composites for Construction, 21(1), 04016057.

Sagar, R. V. and Prasad, B. K. R., (2013). "Laboratory investigations on cracking in reinforced concrete 
beams using on-line acoustic emission monitoring technique." Journal of Civil Structural Health Monitoring, 3(3), 169-186.

Sagar, R. V. and Rao, M. V. M. S., (2015). "Acoustic emission during flexural deformation of reinforced concrete under incremental cyclic loading." Journal of Testing and Evaluation, 44(6), 2182-2198.

Shahidan, S., Pulin, R., Bunnori, N. M. and Holford, K. M., (2013). "Damage classification in reinforced concrete beam by acoustic emission signal analysis." Construction and Building Materials, 45, 78-86.

Sohn, H., Farrar, C. R., Hemez, F. M., Shunk, D. D., Stinemates, D. W., Nadler, B. R. and Czarnecki, J. J.,
(2004). "A review of structural health monitoring literature: 1996-2001, (LA-13976-MS)" USA: Los Alamos National Laboratory.

Torres, B., Payá-Zaforteza, I., Calderón, P. A. and Adam, J. M., (2011). "Analysis of the strain transfer in a new FBG sensor for structural health monitoring." Engineering Structures, 33(2), 539-548.

Vaghefi, K., Oats, R. C., Harris, D. K., Ahlborn, T. M., Brooks, C. N., Endsley, K. A., Roussi, C., Shuchman, R., Burns, J. W. and Dobson, R., (2011). "Evaluation of commercially available remote sensors for highway bridge condition assessment." Journal of Bridge Engineering, 17(6), 886-895. 